\title{
Target Orientation Effects on Movement Time in Rapid Aiming Tasks
}

\author{
Yugang Zhang, Bifeng Song, and Wensheng Min \\ School of Aeronautics, Northwestern Polytechnical University, Xi' an, Shaanxi 710072, China \\ zhang_yu9999@163.com
}

\begin{abstract}
An attempt was made to investigate the effect of the target orientation on pointing performance. An experiment was accomplished in which 10 subjects performed three-dimensional aiming tasks under the manipulation of target orientation, distance to target and direction to target. Results show that target orientation affects the duration of three-dimensional movements significantly. As a result, the conventional movement model did not satisfactorily explain the variance in the movement times produced. The conventional model was employed by incorporating an oriented parameter into the model. The modified model was shown to better fit the data than the conventional model, in terms of $r^{2}$ between the measured movement time and the value predicted by model fit.
\end{abstract}

Keywords: Human movement, Pointing performance, Fitts' Law, Index of difficulty, Target orientation.

\section{Introduction}

Modern human computer interfaces (for example, the touch screen, and the threedimensional display) are becoming much more popular so it is important to determine whether current movement model can provide useful predictions for these interfaces as well $[1,13]$. Since the famous speed-accuracy model of human movement, Fitts' law, was developed, many researchers have verified it over a wide range of conditions [11] and applied in primarily two ways, as a predictive model, and as a means of the comparison and evaluation of pointing devices [7,12].

Fitts' law as originally is a one-dimensional model of human movement. It predicts the movement time $M T$ to select a target of width $W$ and distance (or amplitude) $A$ from the starting point. MacKenzie and Buxton [8] extended it to two-dimensional tasks. They applied target amplitude $A$, target width $W$, target height $H$, and approach angle $\theta_{\mathrm{A}}$ to their two-dimensional model. Then Accot and Zhai [2] further investigated bivariate pointing based on Fitts' law model. They focused on the effect of target shape (target width and height ratio) on pointing performance. Moreover, Murata and Iwase [10] extended Fitts' law to three-dimensional pointing tasks and incorporated a directional parameter into the model. In contrast, Grossman and Balakrishnan [5] proposed a new model that describes pointing at trivariate targets. In addition, 
researchers had studied many other factors, such as trade off of speed and accuracy [4], target scale [6]. However, previous records have failed to consider target orientation. Thus, the results are controversial when apply typical model to three-dimensional pointing tasks. Uncertainties still exist.

The target orientation means, in real world, the target object (e.g., a button, switch, even "iconic" menu) is lying on a surface and cannot exist solely. The plane of target and the plane of starting point present an angle, which we define as the "target orientation" (see Fig. 1 for an illustration).।

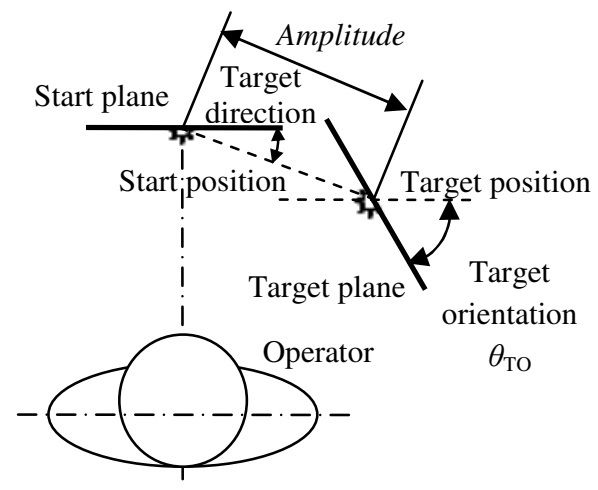

Fig. 1. Sample operation condition

The present study concerns the effect of the target orientation on pointing performance. The control of the forces over the amplitude becomes far complicated with a present of the target orientation. The task is a three-dimensional pointing task and exacter muscular force is required, leading to more variable movement trajectories and, hence, more variable pointing times [14]. Based on these insights into aiming movements, we argued that the pointing movements studied in the present experiment would be sensitive to the effects of target orientation. Fitts' law would be out of action.

In this paper, we studied to examine this hypothesis and to model operator performance in the most fundamental interaction task - pointing - in an experiment where the target orientation varied. To anticipate, we realized this goal as follows.

First, we used the conventional Fitts' model to predict movement time data collected in a pointing task under the manipulation of target orientation, distance to target and direction to target. The fit was suboptimal due to the variance present in the data and the dependency of movement time on target orientation. Second, based on these results, a modified three-dimensional model of Fitts' law was proposed, which was shown to describe the data better than the conventional Fitts' model. Third, we investigated the effects of the factors identified. Finally, we concluded by discussing implications for user interface design. 


\section{Methods}

We will provide in this section experiment method which are necessary for the understanding of subsequent results.

\subsection{Subjects}

Ten Northwestern Polytechnical University healthy male postgraduates (24-26 years of age) participated in the experiment. The subjects were all right-handed and inexperienced with regard to the purpose of the experiment.

\subsection{Apparatus}

The experiment was conducted on LenovoR PC equipped with two 21.5" touch screen LCD monitor $(47.8 \mathrm{~cm} \times 27 \mathrm{~cm}$ visual area, $1024 \times 768$ pixels, 96 dpi resolution). The two monitor was placed vertically on the same shelf (see Fig. 2). One of them showed the starting point and another showed the target point.

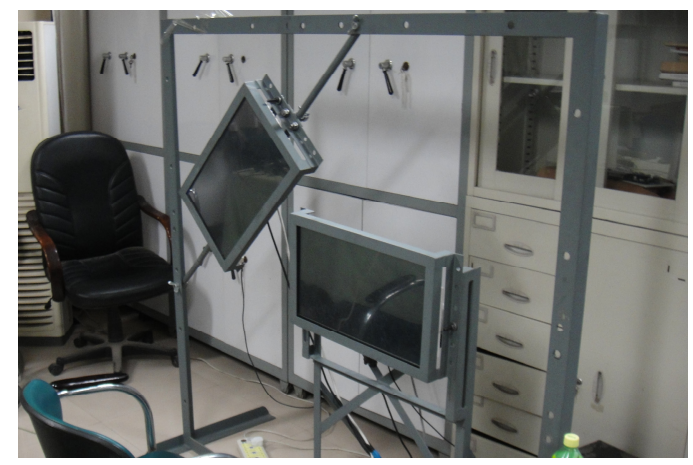

Fig. 2. Physical setup for a pointing task

\subsection{Task}

The task's paradigm is discrete task. The starting point was placed on the median sagittal plane of the subject, which was about $60 \mathrm{~cm}$ away in front of the subject. The subject was required to place his right index finger at the starting point before the experimenter gave him the signal to start the movement. The subject's task was to point with the right index finger to the target specified by the experimenter.

A two-dimensional circle was used as the target to equalize the distance between the starting point and the target for each orientation condition with equal values of target size and distance. In this study, three-dimensional pointing means that the 
movement of the pointer (tip of the index finger) is performed in a three-dimensional space and measured along three axes.

\subsection{Design and Procedure}

A within-subject factorial design with repeated measures was used. The independent variables were the distance to target (three levels: $150,300,450 \mathrm{~mm}$ ), target orientation (four levels: $0^{\circ}, 30^{\circ}, 60^{\circ}, 90^{\circ}$ ) and direction to target (two levels: $0^{\circ}, 30^{\circ}$ ). The target width is $18 \mathrm{~mm}$. Dependent variables were movement time $(M T)$. The error rate was controlled at about $4 \%$. There were 24 different combinations in total.

The experiment included two sessions: a practice session, to allow participants to get used to the task and conditions, and a data-collection session, wherein participants tested the 24 different combinations in certain order. Within each condition, participants performed 20 trials.

After having signed an information consent statement, each subject was tested. The subjects were instructed to carry out the task as accurately and as quickly as possible. The time when the fingertip began to leave from the screen of the starting point was used as a criterion for movement onset. The criterion indicating the end of the movement (trial) was the time when the tip of the index finger reached the screen of the target point. The movement (pointing) time was obtained using developed test software. If the coordinate was within or on the target circle, then the trial was regarded as successful. All other cases were designated as error trials.

\section{$3 \quad$ Results}

Outliers were got rid of based on mean movement time and accuracy - defined as distance between the click point and the target center. Any data further than 3 standard deviations away from its condition's mean (by MT) was removed. $0.9 \%$ of the data were removed as outliers.

\subsection{Movement Time Analysis}

Figure 3 shows how mean movement time changes as a function of the set of amplitude tested in our experiment, while all other factors are balanced. In the figure, " $0{ }^{\circ}$ Motion" means the direction to target is $0^{\circ}$ and " $30^{\circ}$ Motion" means $30^{\circ}$. Immediately noticeable is that the mean movement time (averaged over all orientation conditions and all subjects) generally increases with the increasing distance to target. The movement time of $30^{\circ}$ target direction is shorter than that of $0^{\circ}$ target direction. Analysis of variance showed that the independent variable $A\left(F_{2,18}=1862, p<.01\right)$ has a significant effect on $M T$. This is consistent with the findings of previous studies [3]. 


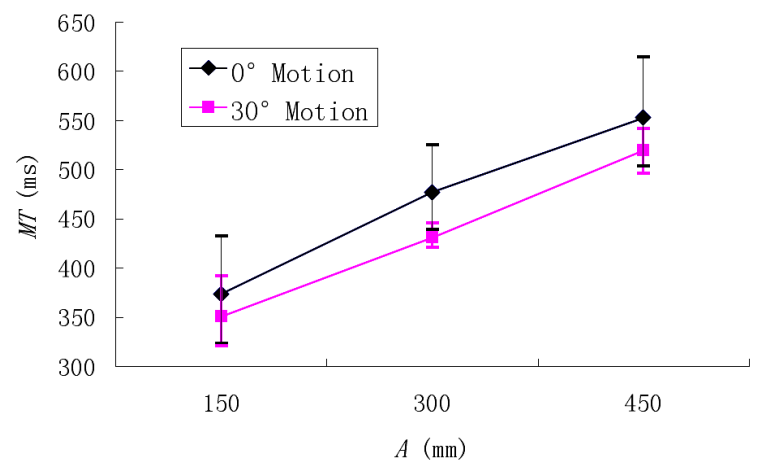

Fig. 3. Influence of target relative position on MT

\subsection{Fitting the Data to the Conventional Fitts' Model}

First, the data were modeled using the following conventional Fitts' model [9]:

$$
M T=a+b \log _{2}(A / W+1.0)
$$

Where $M T$ represents the time to move the right index finger from the starting point to the target, and $A$ and $W$ are the distance from the starting point to the target and the size (diameter) of the target, respectively. The term $\log _{2}(A / W+1.0)$ is the index of difficulty carrying the unit of bits. Finally, the parameters $a$ and $b$ are empirical constants to be determined through linear regression.

The mean $M T$ was calculated for each index of difficulty, pooled over all orientation conditions and subjects. The $r^{2}$ of the linear regression between mean $M T$ and index of difficulty was 0.789 (see Fig. 4). On the basis of this relatively poor fit it can be concluded that there is still substantial room for improving upon the conventional Fitts' model when it comes to the description of three-dimensional movements.

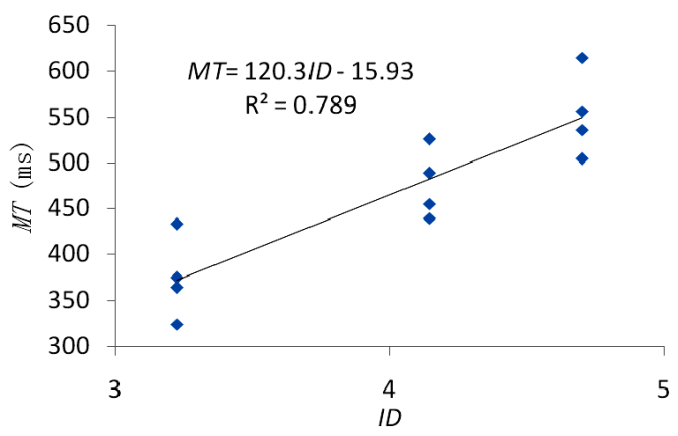

Fig. 4. Relationship between index of difficulty (ID) and movement time 


\subsection{Modifying Fitts' Law to a Three-Dimensional Pointing Task}

To find a meaningful extension of the conventional Fitts' model to three-dimensional pointing movements, we went on to examine the expected relationship between movement time and target orientation.

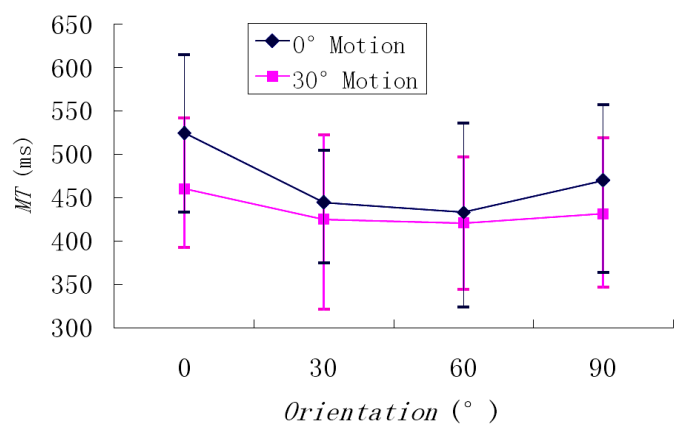

Fig. 5. Influence of target orientation on MT

Figure 5 shows how the mean movement time $M T$ (averaged over all amplitude conditions and all subjects) varied across the four levels of target orientation. The tendency likes a "bowl" shape. The subjective impression from this figure that $M T$ depended on orientation was confirmed in a one-way ANOVA, which revealed a significant main effect of $\theta_{\mathrm{TO}}\left(F_{3,27}=167.198, p<.01\right)$. To identify the source of this statistically significant effect, a multiple comparison post hoc test was performed (i.e., Least Significant Difference test) using a conservative significance level of $p<.01$. On this test, all the comparisons were significant. These results indicate the presence of a systematic relationship between movement time and the target orientation. These imply that a model considering orientation will lead to a better performance model than the conventional Fitts' model. Therefore, we judged that the target orientation could be taken into account by incorporating $\theta_{\mathrm{TO}}$ into the $I D$ in Eq. (1). Based on the discussion, the $I D$ was revised using the following formula:

$$
I D_{T O}=\log _{2}(A / W+1.0)-c \sin \left(2 \times \theta_{T O}\right)
$$

Where $c$ is an arbitrary constant to be determined through linear regression.

For several values of $c$, the relationship between $I D_{\mathrm{TO}}$ and movement time $M T$ was established by means of linear regression. However, the target orientation seems to be an important factor in performance modeling, especially the modeling of threedimensional pointing tasks. Figure 6 shows the $r^{2}$ for the data in Figure 4 as a function of $c$. The highest $r^{2}(0.913)$ was found for $c=0.5$. The fit to the experimental data was improved by using the index of difficulty $I D_{\mathrm{TO}}$, which incorporates the effect of orientation on $M T$, and by using the value of $c$ producing the highest $r^{2}$ (Fig. 7). The optimal values of $c$ differed for the experimental data of the individual subjects. 


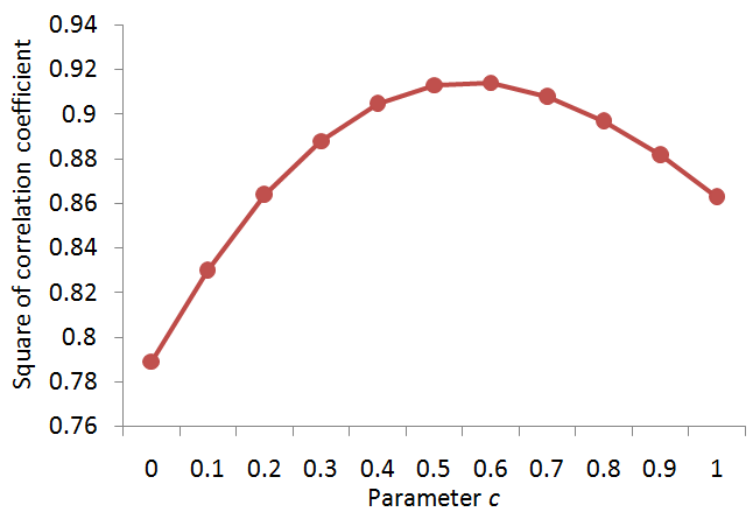

Fig. 6. Squared correlation coefficients as a function parameter $\mathrm{c}$ for fitting the data

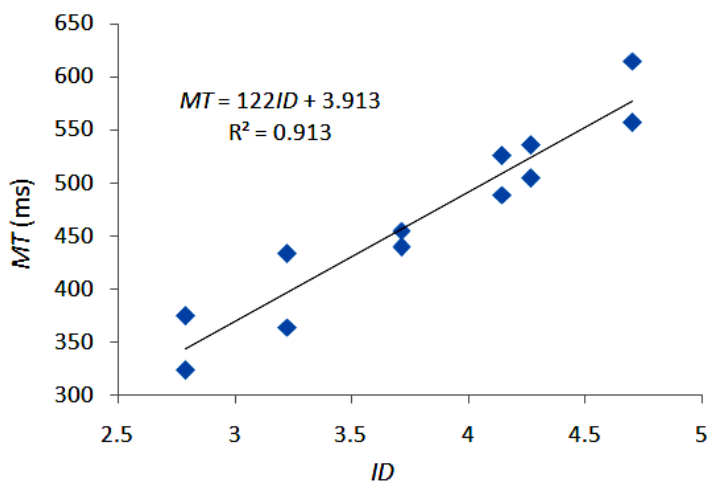

Fig. 7. Movement time as a function of the ID in the modified Fitts' model $(c=0.5)$

The fit to the obtained movement times was better when extended threedimensional modeling was applied. A one-way (modeling method: conventional Fitts' law vs. extended three-dimensional modeling) ANOVA as used for the $r^{2}$ values showed that the difference was significant $\left(F_{1,9}=53.685, p<.01\right)$. Collectively, these results clearly indicate that the modified model of Fitts' law better predicts the duration of three-dimensional (pointing) movements than the conventional Fitts' model.

\section{Conclusions}

The result in Figure 4 and Figure 7 illustrate an issue with traditional applications of Fitts' law to Three-dimensional Pointing Tasks. In the present experiment, movement time was affected significantly by target orientation (cf. Fig. 5). A tendency of "bowl" shape was found. It is not the shortest movement time when target object is lying on the same surface (target orientation is $0^{\circ}$ ) of starting point. 
Thus, the conventional Fitts' model cannot adequately explain the variance in movement time in a real-world three-dimensional pointing task, as it does not take the target orientation into account (cf. Fig. 4). We can come to a conclusion that the interpretation of target orientation and the formulation used in the calculation of a task's index of difficulty play a critical role in the accuracy of the model.

Our study investigated how target orientation affect selection performance, and the results can provide us with significant guidelines on the layout design of car, airplane, and manipulator device, i.e. how items such as widgets, menus, and other objects should be sized and positioned in 3D layout.

In the present research, four levels of target orientation were employed. In future research, more graded levels of orientation will be used in order to confirm the reproducibility of our results. In other words, the relationship between the movement time and the target orientation must be confirmed using an experimental paradigm with more than four levels of target orientation. Furthermore, the subjects in the present study were all right-handed. Future research may investigate modeling that involves right-handed subjects performing pointing tasks with their left hands and left-handed subjects participating in the experiments. Finally, in the present experiment the pointing was conducted, for the sake of simplicity, by using a two-dimensional target in a three-dimensional space. In future research, pointing movements to three-dimensional targets should be examined, requiring a method for defining the target size for a sphere and bringing in depth to the task.

Acknowledgements. This work is supported by National Basic Research Program of China (973 Program), 2010 CB734101 and 2012 undergraduate final paper support program of Northwestern Polytechnical University.

\section{References}

1. Abdulin, E.: Using the Keystroke-Level Model for Designing User Interface on MiddleSized Touch Screens. In: Proceedings of CHI 2011 Conference on Text Entry \& Typing, Vancouver, BC, Canada, May 7-12, pp. 673-686 (2011)

2. Accot, J., Zhai, S.: Refining Fitts' Law Models for Bivariate Pointing. In: Proceedings of CHI 2003 Conference on Human Factors in Computing Systems, Ft. Lauderdale, Florida, USA, April 5-10, pp. 193-200 (2003)

3. Boritz, J., Booth, K.S., Cowan, W.B.: Fitts' law studies of directional mouse movement. In: Proceedings of Graphics Interface, Toronto, pp. 216-223 (1991)

4. Dean, M., Wu, S.-W., Maloney, L.T.: Trading off speed and accuracy in rapid, goal-directed movements. Journal of Vision 7(5),10, 1-12 (2007)

5. Grossman, T., Balakrishnan, R.: Pointing at Trivariate Targets in 3D Environments. In: Proceedings of CHI 2004 Conference on Human Factors in Computing Systems, Vienna, Austria, April 24-29, pp. 447-454 (2004)

6. Guiard, Y.: The Problem of Consistency in the Design of Fitts' Law Experiments: Consider either Target Distance and Width or Movement Form and Scale. In: Proceedings of the 27th International Conference on Human Factor in Computing Systems, Boston, Massachusetts, USA, April 4-9, pp. 1809-1818 (2009) 
7. MacKenzie, I.S., Isokoski, P.: Fitts' Throughput and the Speed-Accuracy Tradeoff. In: Proceedings of CHI 2008 Conference on Human Factors in Computing Systems, Florence, Italy, April 5-10, pp. 1633-1636 (2008)

8. MacKenzie, I.S., Buxton, W.: Extending Fitts' Law to Two-dimensional Tasks. In: Proceedings of CHI 1992 Conference on Human Factors in Computing Systems, New York, USA, May 3-7, pp. 219-226 (1992)

9. MacKenzie, I.S.: A note on the information-theoretic basics for Fitts' law. Journal of Motor Behavior 21, 323-330 (1989)

10. Murata, A., Iwase, H.: Extending Fitts' law to a three-dimensional pointing task. Human Movement Science 20, 791-805 (2001)

11. Plamondon, R., Alimi, A.M.: Speed/accuracy trade-offs in target-directed movements. Behavioural and Brain Sciences 20, 279-349 (1997)

12. Soukoreff, R.W., MacKenzie, I.S.: Towards a standard for pointing device evaluation, perspectives on 27 years of Fitts' law research in HCI. Int. J. Human-Computer Studies 61, $751-789$ (2004)

13. Teather, R.J., Stuerzlinger, W.: Target pointing in 3D user interfaces. In: Proceedings of the Graphics Interface Poster Session (GI 2010), Ottawa, Ontario, Canada, May 31-June 2, pp. 20-21 (2010)

14. VanGalen, G.P., DeJong, W.P.: Fitts' law as the outcome of a dynamic noise filtering model of motor control. Human Movement Science 14, 539-571 (1995) 